\title{
Analysis and research on precise control mode of outdoor substation noise
}

\author{
Luwen $\mathrm{Xu}^{1}$, Bo Yang ${ }^{1, *}$, Tao Yang ${ }^{1}$, Anxin Zou ${ }^{1}$ and Lin Zhang ${ }^{2}$ \\ ${ }^{1}$ State Grid Chongqing Electric Power Research Institute, Chongqing, China \\ ${ }^{2}$ School of Marine Science and Technology, Northwestern Polytechnical University, Xi'an, China
}

\begin{abstract}
As people pay more and more attention to the environmental noise problem of substations, it is an important environmental protection work to carry out noise control of over-subjected substation. According to the lack of strict scientific argumentation, poor engineering control effect and high project cost, the paper proposes an accurate control model for outdoor substation noise. Taking the actual substation as an example, with the development of the substation environmental noise analysis system software SENCS, through six steps of data collection, model verification, pre-treatment analysis, optimization of governance plan and analysis of management effects, it is proposed the model of precise control of outdoor substation, which can play an important guiding role in outdoor substation noise control and new substation planning and design..
\end{abstract}

\section{Introduction}

In recent years, with the increasing awareness of environmental protection among citizens, environmental complaints about noise nuisance in substations have occurred frequently. On the other hand, the state pays more and more attention to environmental protection work, and has increased penalties for some violations of environmental protection regulations, which has brought great pressure to the environmental protection work of power enterprises. State Grid Corporation of China attaches great importance to the disturbance of substation noise, and has specially formulated 《 the Work Plan for Special Action for Substation (Converter Station) Noise Over-Standard Governance》 to guide the development of substation noise control work, and has achieved certain achievements. However, some problems are still exposed in the treatment work, and some projects still fail to meet the requirements of environmental protection standards after treatment, and there are re-complaints or rework; There are also some noise control projects that blindly install noise reduction facilities on a large scale because they can't accurately locate the crux of exceeding the standard, which leads to the false high cost of engineering control. The main reason is the lack of strict scientific proof of the governance scheme, especially in the Pre-simulation calculation link, which is simple and rough, and lacks multi-dimensional in-depth analysis and research process. The goal of substation noise control is to ensure that both the station boundary and the sensitive target can meet the relevant environmental protection standards, but the standard grades reached by the two may be the same or different, and the standard grades reached by the station boundary in all directions of the substation are also different [1-2]. At present, there are two main noise control methods in outdoor substations. The first one is to reduce the noise emission level by overhauling or replacing the noise source equipment. The second one is installing sound absorption and insulation barriers to cut off noise transmission paths, which ensures that substation boundaries or sensitive targets meet environmental protection standards [3-8]. Among them, reducing noise through overhaul is the most economical and practical treatment method, which should be considered as a priority. If the treatment goal is still not reached, the method of adopting sound barrier or replacing low-noise equipment should be considered. The choice between the two needs to be weighed by economic and technical comparison, and one or both of them may be chosen.

At present, in outdoor substation noise control project, the most common control method is to install noise barriers, including single barrier or multi-barriers combination. Generally speaking, under the condition of not affecting the normal operation and maintenance of equipment, the closer the sound barrier is installed to the sound source, the better. Sometimes, the buildings in the station should be reasonably used as sound insulation bodies to reduce the amount of sound barrier and improve the noise control effect. Therefore, in order to achieve the goal of accurate noise control, it is necessary to master the spatial distribution of noise before control and the noise transmission route [9-10], so that the size and position of the noise barrier can be designed in a targeted way to ensure the realization of the noise control goal of the substation and effectively control the project cost.

\footnotetext{
* Corresponding author: hdyb2009@sina.cn
} 
Therefore, taking the actual substation as an example, this paper carries out the model analysis and research of accurate noise control, aiming to provide technical reference for the treatment of noise exceeding standard in outdoor substation.

\section{Theory basis of precision control of outdoor Substation noise}

There are many sound sources in outdoor substation, the most important one of which is the transformer. In addition, there are reactors and cooling fans, etc. The noise generated by these sound sources will be continuously attenuated by atmospheric absorption, geometric scattering, ground effect and obstruction of obstacles in the process of spatial propagation from near field to far field.[11-12] The octave band of noise at a certain measuring point in substation environment can be calculated by:

$$
\begin{aligned}
& \mathrm{L}_{\text {oct }}(r)=\mathrm{L}_{\text {oct ref }}(s) \\
& -\left(\mathrm{A}_{\text {oct div }}+\mathrm{A}_{\text {oct bar }}+\mathrm{A}_{\text {oct atm }}+\mathrm{A}_{\text {oct gr }}+\mathrm{A}_{\text {oct exc }}\right)
\end{aligned}
$$

Where $\mathrm{L}_{\text {oct ref }}(s)$ is the power level of octave band sound generated by equipment sound source; $\mathrm{A}_{\text {octdiv }}$ is the attenuation of octave band sound pressure level caused by geometric divergence; $\mathrm{A}_{\text {octbar }}$ is the attenuation of octave band sound pressure level caused by barrier (sound barrier or building); $\mathrm{A}_{\text {octatm }}$ is the attenuation of octave band sound pressure level caused by atmospheric absorption; $\mathrm{A}_{\text {octgr }}$ is the attenuation of sound pressure level in octave band caused by ground effect and $A_{\text {octexc }}$ is the attenuation of octave band sound pressure level caused by other effects. These variables can be calculated as follows:

$$
\begin{gathered}
\mathrm{A}_{\text {oct div }}=20 \lg (r) \\
\mathrm{A}_{\text {oct atm }}=\alpha(r-1) / 100 \\
\mathrm{~A}_{\text {oct gr }}=4.8-\left(2 h_{\mathrm{m}} / r\right) \cdot[17+(300 / r)] \\
\mathrm{A}_{\text {oct bar }}=-10 \lg \left[\frac{1}{3+20 N_{1}}+\frac{1}{3+20 N_{2}}+\frac{1}{3+20 N_{3}}\right]
\end{gathered}
$$

where in the above formulas $B$ is the distance between sound source and prediction point $(\mathrm{m}) ; h_{\mathrm{m}}$ is the average height from ground between measuring point and equipment sound source $(\mathrm{m}) ; \alpha$ is the atmospheric attenuation coefficient; $N_{\mathrm{i}}$ is the Fresnel number, $N_{\mathrm{i}}=$ $2 \delta_{\mathrm{i}} / \lambda ; \delta_{\mathrm{i}}$ is the acoustic path difference $(\mathrm{m})$ 。

A schematic diagram of noise generated by $S$ bypassing a single barrier is shown in Fig. 1, and the noise $\delta_{\mathrm{i}}$ at the measuring point $R$ can be calculated as follows:

$$
\delta_{\mathrm{i}}=\mathrm{SO}_{\mathrm{i}}+\mathrm{RO}_{\mathrm{i}}+\mathrm{SR}
$$

where $\mathrm{SO}_{\mathrm{i}}, \mathrm{RO}_{\mathrm{i}}$ and $\mathrm{SR}$ represent the noise propagation as illustrated in Fig. 1.

The noise generated by $S$ bypasses two barriers A and $\mathrm{B}$ is shown in Fig. 2. The noise $\delta_{\mathrm{i}}$ at the prediction point $R$ can be calculated as follows:

$$
\delta=\sqrt{\left(d_{\mathrm{ss}}+d_{\mathrm{sr}}+e\right)^{2}+a^{2}}-d
$$

where $a$ is the projection length (m) of SR connecting line in the projection direction of Fig. 2.

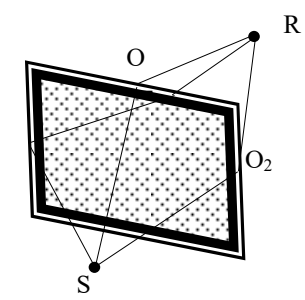

Fig. 1. Diffraction of single barrier



Fig. 2. Diffraction of double barriers

In engineering practice, it is often not a single sound source, so it is necessary to calculate the sound source synthesis, that is, synthesize the noise generated by each sound source in the same frequency band at the prediction point, and then calculate the total sound level of the prediction point through the total value of each frequency band after synthesis [12]. The calculation formulas are shown in Eq. (8) and Eq. (9).

$$
\mathrm{L}_{\mathrm{Ri}}=10 \lg \left(\sum_{\mathrm{k}=1}^{\mathrm{n}} 10^{0.1\left(\mathrm{~L}_{\mathrm{Rk}}-\Delta \mathrm{L}_{\mathrm{k}}\right)}\right)
$$

Where $\mathrm{L}_{\mathrm{Ri}}$ is the Noise calculation value of the $i$ th sound source at the prediction poin $R ; \mathrm{K}$ is the one of the frequency band from 1 to $n ; \mathrm{L}_{\mathrm{Rk}}$ is the Noise value of the $k$ th frequency band; $\Delta \mathrm{L}_{\mathrm{k}}$ is the correction coefficient of the $k$ th frequency band.

Total sound level of measuring points synthesized by each frequency band:

$$
\mathrm{L}_{\mathrm{R}}=10 \lg \left(\sum_{\mathrm{i}=1}^{\mathrm{m}} 10^{0.1 \mathrm{~L}_{\mathrm{Ri}}}\right)
$$

Similarly, the noise barriers or obstacles often encountered in the path of sound propagation in engineering practice are not single, and the vertical and horizontal layout, staggered height and different shape of the noise barrier. Combined with the spatial relationship between sound sources and measuring points, the noise field is extremely complex, which brings great difficulties to the calculation and modelling of substation noise. Therefore, complex multi-barriers are often simplified in engineering to facilitate calculation. As shown in Fig. 3, it is assumed that the connecting line between the sound source and the prediction point is perpendicular to all sound barriers, and there are four sound barriers A, B, C and D with similar acoustic performance. The simplified calculation methods are different because of the different height changes of the four sound barriers. In Fig. 3(a), B can be directly used as the only barrier, and the three barriers A, C and D can be ignored; In Fig. 3(b), A and D are calculated as double barriers, and $\mathrm{B}$ and $\mathrm{C}$ barriers are ignored; In Fig. 3(c), A, B and C are calculated as three barriers, and two barriers $\mathrm{B}$ and $\mathrm{C}$ are ignored; In Fig. 3(d), A and D are 
calculated as double barriers, and B and C barriers are ignored. When there are more noise barriers, it becomes more and more important to choose reasonable simplified processing, which is beneficial to improve the calculation efficiency.


Fig. 3. Multi-barrier diffraction barriers

\section{Precision control mode of outdoor substation noise}

Taking a $220 \mathrm{kV}$ substation as an example, this paper introduces the accurate noise control mode. The main noise sources of the substation are two main transformers with a capacity of 120MVA. There are main buildings such as control building, electrical room and guard room in the substation, and there are two $30 \mathrm{~m}$ high-rise residential buildings and three $5 \mathrm{~m}$ high small houses outside the substation.

\subsection{Basic Data Collection}

The accuracy and comprehensiveness of basic data collection is the basis of accurate noise control calculation in outdoor substations. The original data of parameters collected include: (1) The plan of substation and its surrounding environment, which includes the location relationship parameters of noise source equipment, buildings and sensitive targets outside the substation; (2) Main noise source parameters, including octave sound power of each main noise source, geometric parameters and spatial position of each noise source, etc. The maximum sound level of equipment with high load should be collected as much as possible during noise source collection; (3) Geometric parameters of length, width and height of buildings, walls and sensitive targets outside the substation; (4) Noise monitoring data of substation boundary and sensitive target; (5) The height parameters and data of the substation and the space outside the substation are accurate, and the sound power zone level of the surrounding environment is located.

\subsection{Establishment and Verification of Calculation Model}

To achieve accurate control of substation noise, the analysis of tool software is indispensable. The Substation
Environment Noise Control System (SENCS) developed by Chongqing Electric Power Research Institute of State Grid is a special analysis software for substation noise control. Using the collected basic data, the noise simulation calculation model of the outdoor substation is established, as shown in Fig. 4.

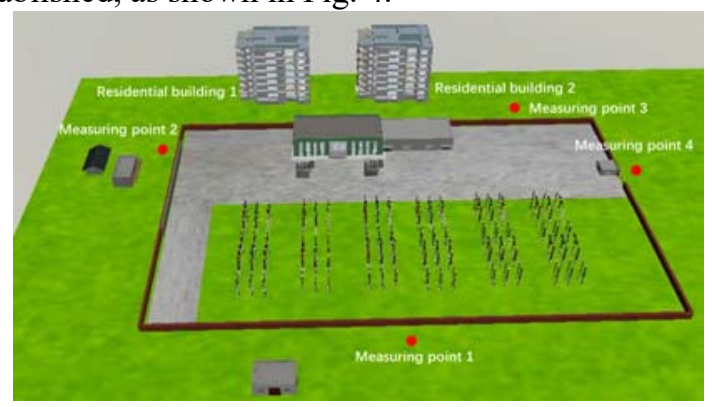

Fig. 4. Substation modeling and measuring point

\subsection{Verification of calculation model}

In order to verify the accuracy of the modeling calculation, it is necessary to use the measured data of the substation to verify. Four measuring points with different heights are arranged around the substation, corresponding to the four measuring points in the simulation model, as shown in Fig. 4. As shown in Tab.1, the predicted value is close to the measured value, which proves that the simulation model and the measured result are correct and can meet the needs of practical engineering analysis. If there is a big gap between them, it is necessary to check the measured data and simulation model to eliminate excessive errors.

Table 1. Comparison of the measuring and calculating value at 4 measuring points

\begin{tabular}{|c|c|c|c|c|}
\hline $\begin{array}{c}\text { Measuring } \\
\text { point }\end{array}$ & Height/m & $\begin{array}{c}\text { Measuring } \\
\text { value/dB }\end{array}$ & $\begin{array}{c}\text { Calculating } \\
\text { value/dB }\end{array}$ & Error/dB \\
\hline 1 & 1.5 & 42.1 & 40.9 & -1.2 \\
\hline 2 & 3.0 & 50.2 & 50.4 & 0.2 \\
\hline 3 & 3.0 & 40.1 & 39.2 & -0.9 \\
\hline 4 & 1.5 & 44.9 & 44.3 & -0.6 \\
\hline
\end{tabular}

\subsection{Noise analysis before substation management}

The purpose of noise simulation, analysis and calculation before noise control in substation is to find out the position and condition of exceeding the standard, and then work out a reasonable control scheme.

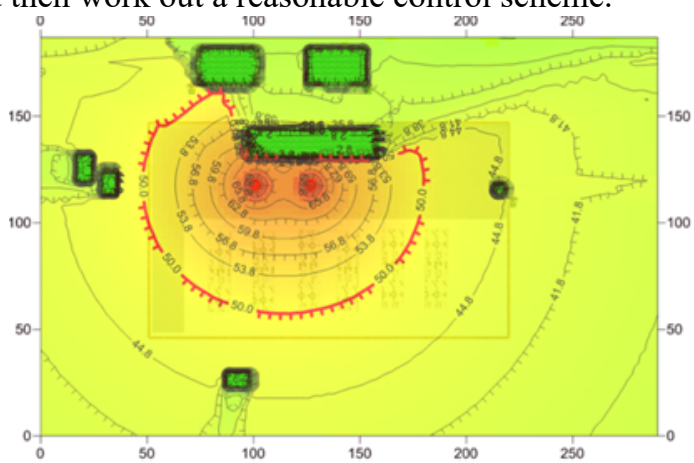

Fig. 5. Noise contour of substation at $3 \mathrm{~m}$ in height 
Fig. 5 is the contour distribution map of $3 \mathrm{~m}$ high noise in substation. It can be seen from this map that some station boundaries on the west side and the station boundaries on the left side of the north side exceed the standard limit of $50 \mathrm{~dB}$, and all other station boundaries meet the standards. Fig. 6 and Fig. 7 are the analysis of the west and north station boundaries, showing the specific location of the station boundary exceeding the standard and the noise level.

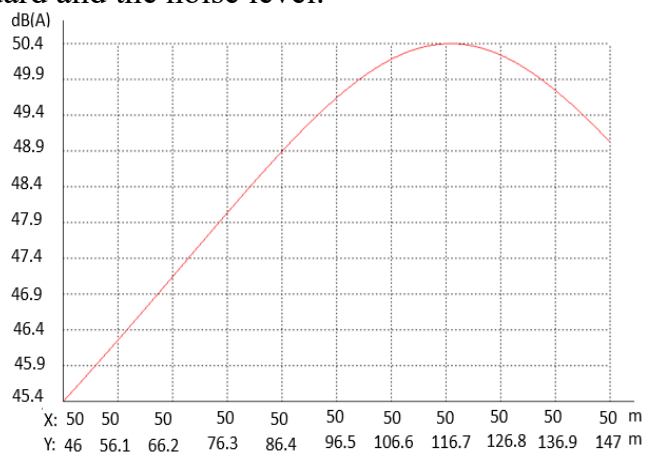

Fig. 6. Noise distribution curve of the west substation

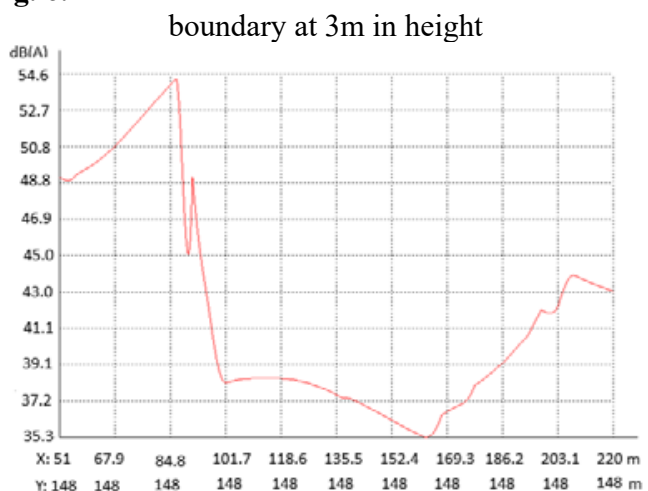

Fig. 7. Noise distribution curve of the north substation boundary at $3 \mathrm{~m}$ in height

Fig. 8 is the elevation noise distribution map of two residential buildings on the north side. The two dashed boxes in the map represent the locations of residential buildings 1 and 2, respectively. Through Fig. 8, we can see the sound and shadow areas left by the control building and the electrical room on the two residential buildings.

It can be seen from Fig. 9 that the $50 \mathrm{~dB}$ isosurface exceeds the standard on the middle section fence, but the two residential buildings outside will not exceed the standard, which is consistent with the analysis of Fig. 5 and Fig. 6; It can be seen from Fig. 10 that the $50 \mathrm{~dB}$ isosurface exceeds the standard in some sections of the fence and two residential buildings, which is consistent with the analysis in Fig. 5 and Fig. 7.



Fig. 8. Noise distribution of two residential buildings on the north side



Fig.9. 3D map of noise exceeded of the west substation

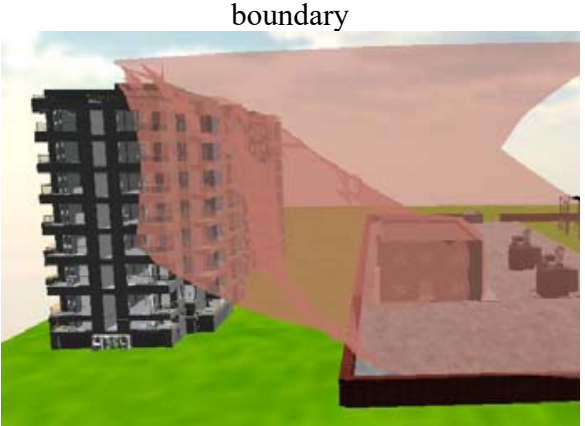

Fig. 10. 3D map of noise exceeded of the north residential buildings and substation boundary

\subsection{Substation Noise Control Scheme is Put Forward}

Through the comprehensive analysis of noise before treatment, it is known that the noise exceeding the standard on the north and west sides needs to be effectively treated by using sound absorption barriers. The general principles of noise reduction by installing sound barriers are as follows: (1) On the premise of not affecting operation and maintenance, sound barriers should be set as close as possible to sound sources; (2) Reasonably use the existing station buildings to form a combined body with the added sound barrier to reduce noise, so as to improve the sound insulation effect and reduce the project cost; (3) The height and length of the sound barrier should be optimized to avoid the problem of high engineering cost. Therefore, in order to control the two houses in the north and the station boundary exceeding the standard, a sound barrier is installed at the front edge of the control roof in combination with the actual situation in the station.

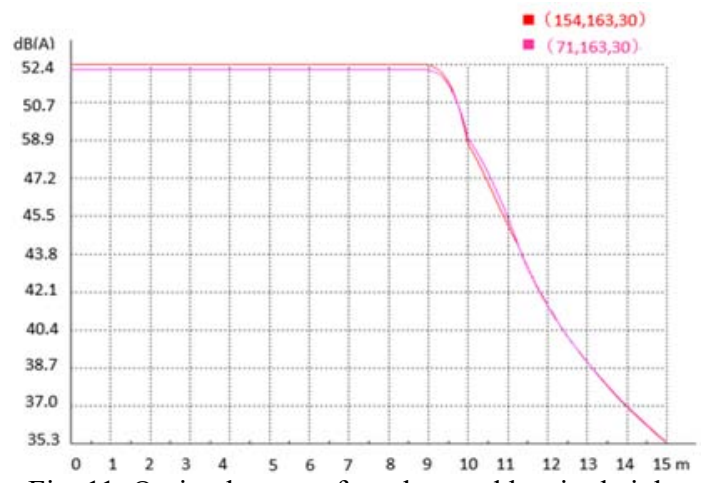

Fig. 11 Optimal curve of north sound barrier height 




Fig. 12 Specific layout and size of sound barrier

Fig. 11 shows the height optimization curve of the sound barrier, in which two curves respectively represent two residential buildings, with the ordinate representing the noise level and the abscissa representing the height of the sound barrier. Therefore, the height is selected to be $11 \mathrm{~m}$, and the height of the sound barrier in the west is calculated to be $4.5 \mathrm{~m}$ by optimization. In this substation treatment scheme, the specific layout and size of the sound barrier are shown in Fig 12.

\subsection{Effect Analysis After Treatment}

Fig .13 shows the effect of noise reaching the standard at the west station boundary after treatment. In the figure, the $50 \mathrm{~dB}$ isosurface is lifted by the sound barrier, which ensures that the station boundary reaches the standard. Fig. 14 shows the effect of reaching the standard between two sensitive buildings on the north side and the station boundary. The distance between the isosurface and the building is sufficient, which proves that the requirements of relevant standards have been met after treatment.

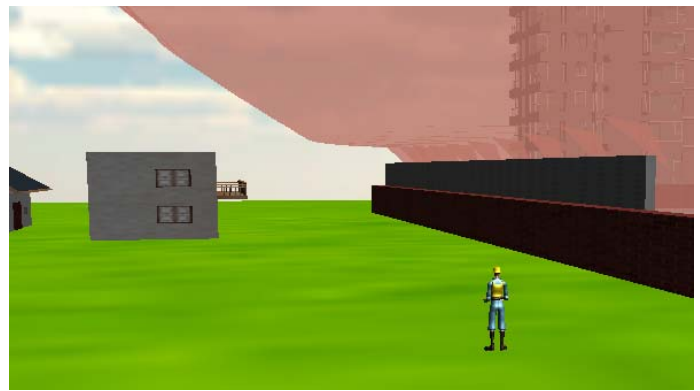

Fig. 13 Noise compliance figure of the west side after governance



Fig. 14 Noise compliance figure of the north side after governance

As the noise of substation noise source equipment will change with the change of load, and may also increase with the increase of operation time, in order to ensure that the substation noise can continue to meet the standard after treatment, the noise value of the sound source obtained by simulation calculation cannot be lower than the value under the maximum load condition, and the sound source must ensure that the boundary conditions of sensitive targets and station boundary noise control have sufficient margin.

\section{Conclusion}

On the basis of theoretical analysis, this paper uses SENCS software to take a $220 \mathrm{kV}$ outdoor substation as an example, from the analysis of basic data to the display of treatment effect, comprehensively and detailly introduces the accurate noise treatment mode of outdoor substation, and draws the following conclusions:

(1) Accurate noise control in outdoor substations is a feasible engineering control mode.

(2) Accurate noise control in outdoor substations requires collecting basic data such as sound source size and position relationship, and must rely on accurate modeling and multi-dimensional simulation calculation and analysis.

(3) The accurate treatment mode of outdoor substations can effectively ensure that sensitive targets and station boundaries meet the standards continuously, and at the same time reduce the project cost, which can provide technical reference for the planning and design of new substations, create great economic and social benefits, and have important popularization and application value.

\section{References}

1. X. F. Wang, W. Li , D. C. Jin, Electric Power Environmental Protection, 33 (2017)

2. C. W. Huang, Guangdong Electric Power, 23 (2010)

3. W. Cai, K. She, X. F. Peng, et al. Smart Power, 47 (2019)

4. H. T. Sun,J. Yang, M. Zhang, et al, Electrical Applications, 38 (2019)

5. H. Liu, J. G. Yin,G. P. Zhang,et al, Guangdong Electric Power, 31 (2018)

6. C. Fan, L. Q. Gong, Z. G. Liu, et al, Smart Grid, 4 (2016)

7. N. Li, D. M. Tian, D. P. Shan, et al, High Voltage Apparatus, 51 (2015)

8. J. G. Zhou, L. H. Li, Y. Du, et al, China Electric Power, 42 (2009)

9. J. Z. Hu, Q. F. Liao,Y. Yang, et al, High Voltage Apparatus, 53 (2017)

10. B. Zhou, C. M. Pei, Y. Ni, et al, High Voltage Technology, 39 (2013)

11. L. W. Xu, A. X. Zou, Noise Control Technology of Substation (China Environment Press, 2018)

12. Y. M. Li, Y. Q. Wang, L. W. Xu, et al, Electric Power Construction, 34 (2013) 